\title{
Corrigendum: L1-associated genomic regions are deleted in somatic cells of the healthy human brain
}

\author{
Jennifer A Erwin, Apuã C M Paquola, Tatjana Singer, Iryna Gallina, Mark Novotny, Carolina Quayle, Tracy A Bedrosian, \\ Francisco I A Alves, Cheyenne R Butcher, Joseph R Herdy, Anindita Sarkar, Roger S Lasken, Alysson R Muotri \& Fred H Gage \\ Nat. Neurosci. 19, 1583-1591 (2016); published online 12 September 2016; corrected after print 13 July 2017
}

In the version of this article initially published, NIH grant T32 CA009370 to F.H.G. was missing from the Acknowledgments. The error has been corrected in the HTML and PDF versions of the article.

\section{Corrigendum: Overlearning hyperstabilizes a skill by rapidly making neurochemical processing inhibitory-dominant}

\author{
Kazuhisa Shibata, Yuka Sasaki, Ji Won Bang, Edward G Walsh, Maro G Machizawa, Masako Tamaki, Li-Hung Chang \& Takeo Watanabe \\ Nat. Neurosci. 20, 470-475 (2017); published online 30 January 2017; corrected after print 18 September 2017
}

In the version of this article initially published, NIH grant R01EY019466 was missing from grants to T.W. in the Acknowledgments. The error has been corrected in the HTML and PDF versions of the article.

\section{Corrigendum: Hormonal gain control of a medial preoptic area social reward circuit}

Jenna A McHenry, James M Otis, Mark A Rossi, J Elliott Robinson, Oksana Kosyk, Noah W Miller, Zoe A McElligott, Evgeny A Budygin, David R Rubinow \& Garret D Stuber

Nat. Neurosci. 20, 449-458 (2017); published online 30 January 2017; corrected after print 30 June 2017

In the version of this article initially published, there were errors in data analysis and presentation. The corrected analysis and presentation do not change the results or interpretation of the data. Asterisk definitions have also been added for clarity as noted below. Changes with respect to the number of subjects reflect errors in reporting only and did not affect the data analysis. The percentages in Figure $1 \mathrm{~b}$ originally reported as 43,3 , 18 and $36 \%$ have been changed to 43, 1, 19 and 37\%, respectively. In the first paragraph of the Results, "97\% of Nts-labeled cells colocalizing with VTA beads" has been changed to "96\% of Nts-labeled cells colocalizing with VTA beads" and "this subpopulation comprised $33 \%$ of all mPOA VTA-projecting neurons" has been changed to "this subpopulation comprised 35\% of all mPOA VTA-projecting neurons." In the legend for Figure 2 a, the $n$ value originally reported as 9 has been changed to 9 and 10, and asterisks have been added to read ${ }^{* *} P=0.0006$. In Figure 3 a, points were misplotted as a result of an error in data analysis. The graph has been replaced. The values originally reported in the legend as $t_{5}=2.82, P$ $=0.0368$ have been changed to $t_{5}=5.85,{ }^{*} P=0.0021$. In the Figure $3 \mathrm{~d}$ legend, the value originally reported as $n=52$ cells has been changed to 51. In Figure 3e, the percentage in the male E2 pie chart for excited neurons has been changed from 24 to $23 \%$. Figure $5 \mathrm{~b}$ originally contained duplicate example traces of calcium transients that were supposed to be taken from three individual neurons; new traces have been supplied. In Figure $5 c, d$, the asterisks have been changed from ${ }^{\star * \star}$ to ${ }^{\star * * *}$ and defined in the legend as ${ }^{\star * * *} P<0.0001$, Bonferroni post hoc test, E2 versus pre and E2 versus post. In the Figure $5 \mathrm{~d}$ legend, the value originally reported as $F_{2,252}=13.13$ has been changed to 17.32 . In the Figure $5 \mathrm{f}$ legend, asterisks have been defined as ${ }^{* * *} P<0.001$, Bonferroni post hoc test. In Figure $5 \mathrm{~g}$, the horizontal axis was truncated at 60 , resulting in missing data points; the graph has been replaced. In the Figure $5 \mathrm{~h}$ legend, asterisks have been defined as ${ }^{\star} P<0.05,{ }^{* *} P<0.01$, ${ }^{\star * *} P<0.001$, Bonferroni post hoc test. In the Figure 6 e legend, the degrees of freedom originally reported as $F_{3,30}$ have been changed to $F_{3,27}$. In the Figure $6 \mathrm{~g}$ legend, the value originally reported as eYFP $=6$ mice has been changed to eYFP $=7$ mice. In the Figure $6 \mathrm{~h}$ legend, the values originally reported as $F_{3,30}=$ 9.44, $P=0.0002$ have been changed to $F_{3,24}=15.2, P<0.0001$. In Figure $6 \mathrm{i}, \mathrm{j}$, points were misplotted as a result of an error in data analysis, and error bars plotted as s.d. were misidentified in the legend as s.e.m. The data have been replotted, with error bars representing s.e.m. In the Figure $6 \mathrm{i}$ legend, the values originally reported as $F_{1,8}=23.2$ have been changed to $F_{1,9}=63.1$. Asterisks have been defined at the end of the Figure 6 legend as ${ }^{\star} P<0.05,{ }^{* *} P<0.001,{ }^{* * *} P<0.0001$, Bonferroni post hoc test. The statistically significant differences in Figure $7 \mathrm{~b}$ originally indicated by ${ }^{* *}$ have been changed to ${ }^{* *}$; these have been defined in the legend as ${ }^{\star *} P<0.001$, Bonferroni post hoc test. In Figure $7 \mathrm{e}$, a data point in the E2 group was missing; the graph has been replaced. An asterisk has also been added in the Figure 7e legend to read ${ }^{\star} P=0.016$. In Figure $8 \mathrm{c}$, data were misplotted as a result of errors in analysis. The graph has been replaced, and the statistically significant differences originally indicated by * have been changed to ${ }^{\star *}$. The values originally reported in the legend as $F_{4,24}=4.20, P=0.0112$ have been changed to $F_{4,21}=6.82, P=0.0011$. In Figure $8 \mathrm{~d}$, data were misplotted as a result of an error in analysis. The graph has been replaced, and the values originally reported in the legend as $F_{4,24}=8.33, P=0.0003$ have been changed to $F_{4,21}=6.35, P=0.0016$. In Figure $8 \mathrm{e}$, data were misplotted as a result of an error in analysis. The graph has been replaced, and the values originally reported in the legend as $F_{4,24}=0.60, P=0.6622$ have been changed to $F_{4,21}=1.33, P=0.29$. In Figure 8g, the vertical axis was truncated at 250, resulting in missing data points; the graph has been replaced, and the statistically significant differences originally indicated by ${ }^{*}$ have been changed to ${ }^{*}$. The values originally reported in the legend as $F_{1,12}=7.92$ as a result of an error in manuscript preparation have been changed to $F_{1,12}=7.15$. In Figure $8 \mathrm{~h}$, the vertical axis was truncated at -0.4 and 0.8 , resulting in missing data points, and data were misplotted as a result of an error in analysis. The graph has been replaced, and the statistically significant differences originally indicated by ${ }^{*}$ have been changed to ${ }^{\star * *}$. The values originally reported in the legend as $F_{1,12}=7.15, P=0.0200$ have been changed to $F_{1,12}=9.8, P=0.009$. In Figure $8 \mathrm{j}$, the vertical axis was truncated at 200, resulting in missing data points. The graph has been replaced, and 